\title{
eLD: entropy-based linkage disequilibrium index between multiallelic sites
}

Yukinori Okada (iD) 1,2

\begin{abstract}
Quantification of linkage disequilibrium (LD) is a critical step in studies investigating human genome variations. Commonly used LD indices such as $r^{2}$ handle LD of biallelic variants for two sites. As shown in a previously introduced $L D$ index of $\varepsilon$, normalized entropy difference of the haplotype frequency between LD and linkage equilibrium (LE) could be utilized to estimate LD of biallelic variants for multiple sites. Here, we developed eLD (entropy-based Linkage Disequilibrium index between multiallelic sites) as publicly available software to calculate $\varepsilon$ of multiallelic variants for two sites. Application of eLD could dissect complex LD structures among multiple HLA genes (e.g., strong LD among HLA-DRB1, HLA-DQA1, and HLA-DQB1 in East Asians). Use of eLD is not restricted to haplotype-based LD; it is also applicable to genotype-based LD. Therefore, eLD enables estimation of trans-regional LD of SNP genotypes at two unlinked loci, such as the nonlinear LD between functional missense variants of ADH1B (rs1229984 [Arg47His]) and ALDH2 (rs671 [Glu504Lys]).
\end{abstract}

Linkage disequilibrium (LD) is defined as the nonrandom distribution of alleles at different $\operatorname{loci}^{1}$. Quantitative assessment of $\mathrm{LD}$ in a population of interest is an important procedure to conduct fine-mapping of causal variants embedded in the disease risk loci identified by genome-wide association studies (GWAS) ${ }^{2}$. Populationspecific features of LD are related to ethnically heterogeneous distributions of single-nucleotide polymorphisms (SNPs) ${ }^{3}$. The most widely used measurements of LD are $r^{2}$ and $D^{\prime}$; both values quantify LD between biallelic variants (i.e., SNPs) for two sites, reflecting nonrandom distributions of four haplotypes consisting of pairwise combinations of the alleles. Specifically, $r^{2}$ can be interpreted as Pearson's correlation measurement $\left(R^{2}\right)$ of allele distributions and is known to be proportional to $\chi^{2}$ values of genotype-phenotype association statistics between two sites $^{1}$. LD values can easily be calculated using publicly available software (e.g., PLINK and vcftools), or using

\footnotetext{
Correspondence: Yukinori Okada (yokada@sg.med.osaka-u.ac.jp)

'Department of Statistical Genetics, Osaka University Graduate School of Medicine, Suita 565-0871, Japan

${ }^{2}$ Laboratory of Statistical Immunology, Immunology Frontier Research Center (WPI-IFReC), Osaka University, Suita 565-0871, Japan
}

downloaded pre-calculated values from websites (e.g., HaploReg and LocusZoom).

Nothnagel et al. ${ }^{4}$ previously demonstrated that $r^{2}$ can also be interpreted as normalized entropy in haplotype frequencies, and introduced a novel LD index named $\varepsilon$ (see definition in Supplementary Information). $\varepsilon$ represents the normalized entropy difference of the haplotype frequencies between LD and those expected under the null hypothesis of no LD (i.e., linkage equilibrium [LE]). The value of $\varepsilon$ ranges between 0 and 1 , with larger values indicating stronger LD. Application of $\varepsilon$ enabled LD quantification of biallelic variants for multiple sites (Fig. 1$)^{4}$, which was effective in selecting tag SNPs free from ambiguous definitions of LD blocks in an unbiased manner ${ }^{5}$.

We have recently extended $\varepsilon$ to further quantify LD of multiallelic variants for two sites as described elsewhere (Fig. 1) $)^{6}$. Here, we developed eLD (entropy-based Linkage Disequilibrium index between multiallelic sites) as publicly available software to calculate the $\varepsilon$ of multiallelic variants for two sites (see the software URL). Various multiallelic variants exist with important clinical impacts in terms of genotype-phenotype associations. Of these, polymorphisms of human leukocyte antigen (HLA) genes 


\section{Bi-allelic variants for two sites $\left(r^{2}\right)$}
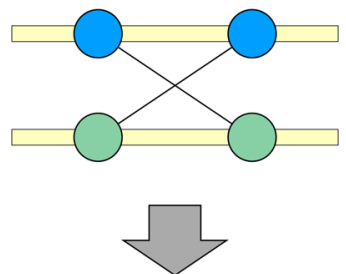

\section{Bi-allelic variants for multiple sites $(\varepsilon)$}

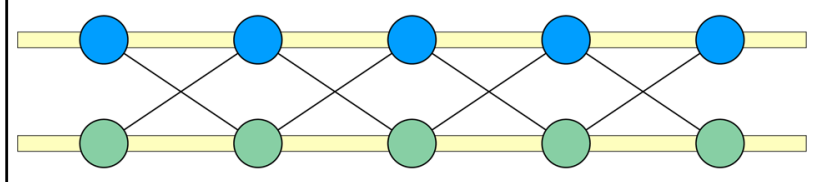

(Nothnagel M et al. Hum Hered 2002)

\section{Multi-allelic variants for two sites $(\varepsilon)$}

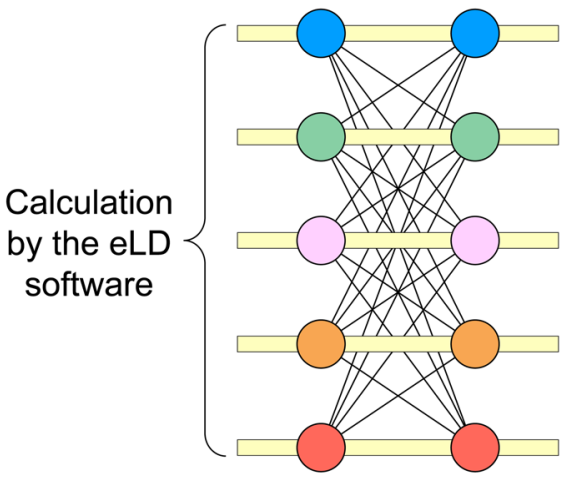

(Okada Y et al. Nat Genet 2015)

Fig. 1 eLD: entropy-based linkage disequilibrium index between multiallelic sites. While $r^{2}$ quantifies LD of biallelic variants for two sites, $\varepsilon$ quantifies LD of biallelic variants for multiple sites ${ }^{4}$ or multiallelic variants for two sites $^{6}$. We developed eLD (entropy-based Linkage Disequilibrium index between multiallelic sites) as publicly available software to calculate $\varepsilon$ of multiallelic variants for two sites (see the software URL)

in the major histocompatibility (MHC) locus have a wide spectrum of risk for a variety of human diseases. While elucidation of the complex LD structure of HLA genes has been challenging, application of $\varepsilon$ clearly identified hidden LD relationships among the HLA genes ${ }^{6}$. For example, we observed relatively strong LD between $H L A-C$ and $H L A$ $B$, among $H L A-D R B 1, H L A-D Q A 1$, and $H L A-D Q B 1$, and between HLA-DPA1 and HLA-DPB1 $(\varepsilon>0.15$; calculated using 4-digit classical alleles of a subset of the East Asian subjects $[n=300]$ enrolled in the original studies ${ }^{6,7}$; Fig. 2). Since estimation of the haplotype frequency could be biased when its distribution is sparse, an option to combine the alleles with frequencies lower than the defined threshold (0.05 in default settings) into a single dummy allele is implemented in eLD.
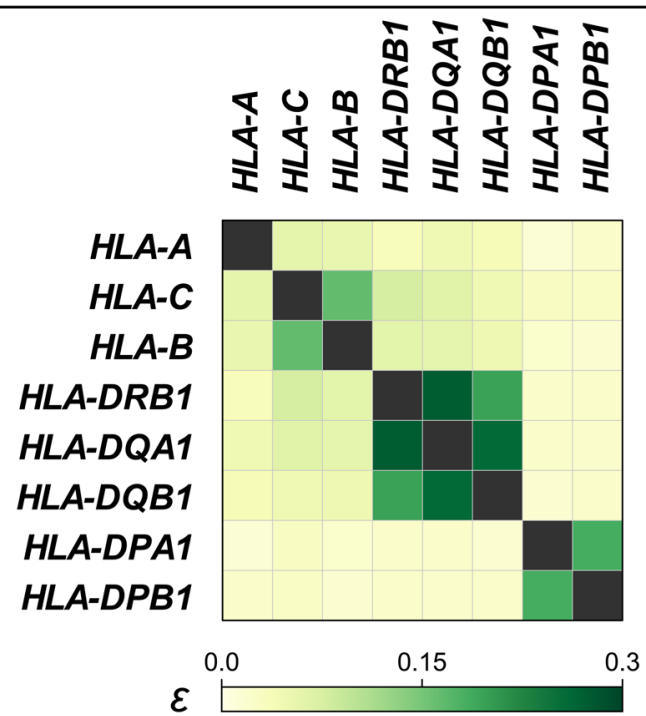

Fig. 2 Pairwise quantification of LD among the classical HLA gene variants. Pairwise LD index of $\varepsilon$ among the 4-digit alleles of the classical HLA genes were evaluated using eLD. Phased HLA alleles were obtained from a subset of the East Asian subjects $(n=300)$ of the original studies, ${ }^{6,7}$. Strong LD between HLA-C and HLA-B, among HLA-DRB1, HLA-DQA1, and HLA-DQB1, and between HLA-DPA1 and HLA-DPB1 was observed $(\varepsilon>0.15)$

One of the novel features of eLD is to empirically estimate a value of $\varepsilon$ in a null hypothesis of LE $\left(=\varepsilon_{-}\right.$NULL $)$. Additionally, it also calculates the $\varepsilon$ actually observed in a given data set ( $=\varepsilon$ Observed). eLD calculates $\varepsilon_{\text {NULL }}$ based on a permutation approach. By randomly shuffling connections of the alleles between the two sites, $\varepsilon$ NULL is estimated as the mean value of $\varepsilon$ obtained in each iteration step $(\times 1000$ iterations in default settings). Since the baseline value of $\varepsilon_{\text {NULL }}$ depends on the number of alleles in each site, calculation of $\varepsilon_{-N U L L}$ as well as $\varepsilon_{-}$Observed would help to evaluate the relative strength of LD relationships at the observed sites.

Another feature of the software is that application of eLD is not restricted to haplotype-based LD; it is also applicable to genotype-based LD. Using eLD, one can estimate LD between loci where phasing of the haplotypes is theoretically difficult. As an illustrative example, we estimated trans-regional LD in two unlinked loci: $A D H 1 B$ at $4 \mathrm{q} 23$ and $A L D H 2$ at $12 \mathrm{q} 24 . A D H 1 B$ and $A L D H 2$ harbor well known functional missense variants at rs1229984 (Arg47His) and rs671 (Glu504Lys), respectively. Both of these SNPs have pleiotropic effects on a number of human complex traits, including dietary habits. Studies investigating natural selection pressure identified strong significant positive selection on these missense variants in Japanese or other East Asian populations, which was closely linked to geographical heterogeneity in allele frequency spectra of these SNPs even within a single population ${ }^{8}$. Here, using eLD, we calculated $\varepsilon$ to estimate trans-regional 


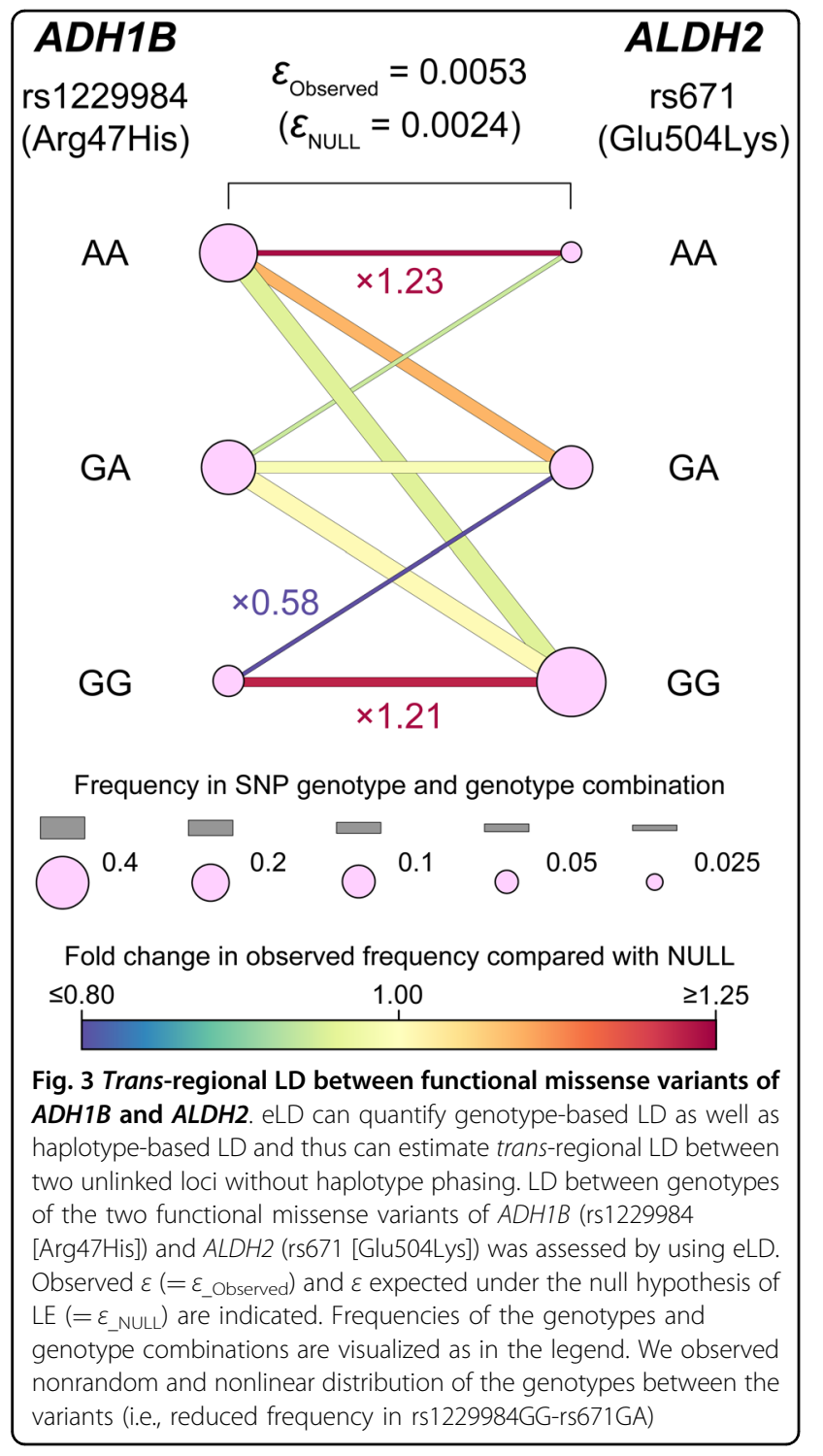

LD between rs1229984 and rs671 (Fig. 3). We obtained genotypes for these SNPs from East Asian subjects within the 1000 Genomes Projects $(n=504$, phase 3 version 5$)$, and found a high $\varepsilon$ observed value $(=0.0053)$ when compared to $\varepsilon_{-N U L L}(=0.0024)$. As expected from natural selection pressure on these variants ${ }^{8}$, rs1229984AArs671AA genotypes and rs1229984GG-rs671GG genotypes had increased frequencies compared to those variants in LE ( $\geq 1.21$-fold), while rs1229984GG-rs671GA genotypes had decreased frequencies (0.58-fold) compared to those variants in LE. While Pearson's correlation between genotypes can also evaluate trans-regional LD, nonlinear relationships of genotypes (such as the reduced frequency of rs1229984GG-rs671GA) would not have been reflected with this measurement.
In summary, we developed software, which we named eLD, that quantifies the entropy-based LD index of $\varepsilon$ in multiallelic variants for two sites, such as LD between highly polymorphic HLA genes. eLD also enables estimation of trans-regional LD of SNP genotypes, such as functional variants of $A D H 1 B$ and $A L D H 2$. We note that normalized entropy has increased the potential to dissect complex dependencies among human genome variations (e.g., Y-chromosomal short tandem repeat [STR] marker selection ${ }^{9}$ ), and development of additional methodology should be warranted.

\section{Software availability}

eLD is freely available at http://www.sg.med.osaka-u.ac.jp/tools.html with example data sets.

\section{Acknowledgements}

We thank Prof. Michael Nothnagel for his thoughtful suggestions. This study was supported by the Japan Society for the Promotion of Science (JSPS) KAKENHI (15H05911) and the Japan Agency for Medical Research and Development (AMED; 18gm6010001 h0003 and 18ek0410041h0002).

\section{Conflict of interest}

The author declares that he has no conflict of interest.

\section{Publisher's note}

Springer Nature remains neutral with regard to jurisdictional claims in published maps and institutional affiliations.

Supplementary information is available for this paper at https://doi.org/ 10.1038/s41439-018-0030-x.

Received: 9 September 2018 Revised: 18 September 2018 Accepted: 18 September 2018.

Published online: 22 October 2018

\section{References}

1. Slatkin, M. Linkage disequilibrium - understanding the evolutionary past and mapping the medical future. Nat. Rev. Genet. 9, 477-485 (2008).

2. Schaid, D. J. From genome-wide associations to candidate causal variants by statistical fine-mapping. Nat. Rev. Genet. 19, 91-504 (2018).

3. Kanai, M. et al. Empirical estimation of genome-wide significance thresholds based on the 1000 Genomes Project data set. J. Hum. Genet. 61, 861-866 (2016).

4. Nothnagel, M. et al. Entropy as a measure for linkage disequilibrium over multilocus haplotype blocks. Hum. Hered. 54, 186-198 (2005).

5. Nothnagel, M. \& Rohde, K. The effect of single-nucleotide polymorphism marker selection on patterns of haplotype blocks and haplotype frequency estimates. Am. J. Hum. Genet. 77, 988-998 (2005).

6. Okada, Y. et al. Construction of a population-specific HLA imputation reference panel and its application to Graves' disease risk in Japanese. Nat. Genet. 47 798-802 (2015).

7. Okada, Y. et al. Risk for ACPA-positive rheumatoid arthritis is driven by shared HLA amino acid polymorphisms in Asian and European populations. Hum. Mol. Genet. 23, 6916-6926 (2014).

8. Okada, Y. et al. Deep whole-genome sequencing reveals recent selection signatures linked to evolution and disease risk of Japanese. Nat. Commun. 9, 1631 (2018).

9. Siegert, S. et al. Shannon's equivocation for forensic Y-STR marker selection. Forensic Sci. Int. Genet. 16, 216-225 (2015). 